Case Report

\title{
Seizure-Associated ST Elevation Myocardial Infarction in Absence of Plaque Rupture
}

\author{
Haytham Mously $\mathbb{D}^{1},{ }^{1}$ Mohammed Wazzan, ${ }^{1}$ Ahmed Z. Alkhathlan, ${ }^{1}$ and Indiresha Iyer ${ }^{2}$ \\ ${ }^{1}$ Department of Internal Medicine, University Hospitals Cleveland Medical Center, Cleveland, OH, USA \\ ${ }^{2}$ Harrington Heart and Vascular Institute, University Hospitals Cleveland Medical Center, Cleveland, OH, USA \\ Correspondence should be addressed to Haytham Mously; Haytham.Mously@uhhospitals.org
}

Received 12 October 2017; Revised 6 March 2018; Accepted 4 April 2018; Published 18 April 2018

Academic Editor: Bruno Megarbane

Copyright (C) 2018 Haytham Mously et al. This is an open access article distributed under the Creative Commons Attribution License, which permits unrestricted use, distribution, and reproduction in any medium, provided the original work is properly cited.

Acute coronary syndrome (ACS) is a very common cause of morbidity and mortality in the U.S. Here, we present a case of acute ST elevation myocardial infarction (STEMI) in the setting of seizure activity. In this rare case, we have data from optical coherence tomography (OCT) that showed no plaque disruption, showing the role of OCT in understanding the pathophysiology of STEMI and providing some ideas for the mechanism of this seizure-induced STEMI.

\section{Introduction}

Acute coronary syndrome (ACS) is a very common cause of morbidity and mortality in the U.S., accounting for approximately 1.5 million of hospital discharges and costing more than 150 billion dollars per year according to the American Heart Association [1]. Moreover, ACS includes unstable angina, non-ST elevation myocardial infarction (NSTEMI), and ST elevation myocardial infarction (STEMI). The pathophysiology includes atherosclerotic plaque rupture or plaque erosion [2]. NSTEMI occurs in the setting of subtotal occlusion of the culprit coronary artery. On the other hand, STEMI occurs due to complete occlusion of the culprit coronary artery [3]. In most cases, the sine qua non of acute myocardial injury is ST elevation on the 12-lead electrocardiogram (ECG). Other noncardiac conditions including acute cerebrovascular events can produce acute ST elevation but are rare $[4,5]$. We present a case of acute ST elevation MI in the setting of seizure activity. In this rare case, we have data from optical coherence tomography that showed no plaque disruption.

\section{Case Presentation}

A 75-year-old African American lady was admitted to the University Hospitals Richmond Medical Center for severe progressive headaches in the setting of hypertensive emergency. Her past medical history included coronary artery bypass graft (CABG) 20 years ago and subsequent multiple prior percutaneous coronary interventions (PCIs) to her right coronary artery (RCA) and left circumflex artery (LCx), and most recently 10 months before with a $2.75 \times 14 \mathrm{~mm}$ Resolute Integrity drug-eluting stent (DES) for an in-stent restenosis of her mid-RCA. Echocardiogram obtained in 2015 revealed reduced ejection fraction of $40-45 \%$ and regional wall motion abnormalities involving apical septal and basal inferior segments and basal and mid inferior septum. She did not have any cardiac symptoms at the time of this admission. Her initial ECG showed sinus rhythm, left ventricular hypertrophy (LVH) by Cornell criteria, and inferior $\mathrm{Q}$ waves (Figure 1). Troponin I was $0.04 \mathrm{ng} / \mathrm{mL}$ (normal $<0.05 \mathrm{ng} / \mathrm{mL}$ ). A noncontrast head CT showed no acute changes. She was admitted for further management. Hydralazine was used to gradually lower her elevated blood pressure. A routine second ECG 13 hours later at 04:53 AM showed similar findings. Serial troponin I values were $0.05 \mathrm{ng} / \mathrm{mL}$ and $0.05 \mathrm{ng} / \mathrm{mL}$ six hours apart. The next morning at 07:35 AM she suffered a tonic-clonic seizure that lasted for 5 minutes. She was treated successfully with lorazepam. An ECG at 08:03 AM after her seizure showed 2.5 to $3 \mathrm{~mm}$ acute ST elevations in leads II, III, and aVF (Figure 2). She was loaded with aspirin and ticagrelor and was started on heparin drip. Her blood 


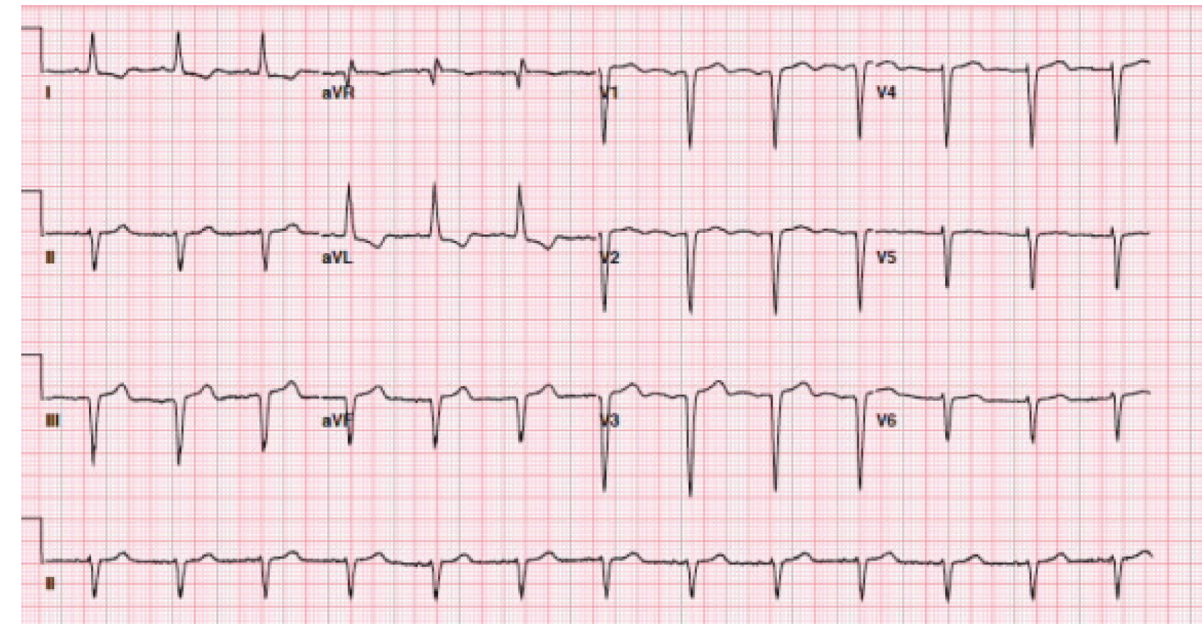

FIGURE 1: Baseline ECG upon admission showing inferior Q waves, nonspecific ST and T wave changes, and evidence of LVH.

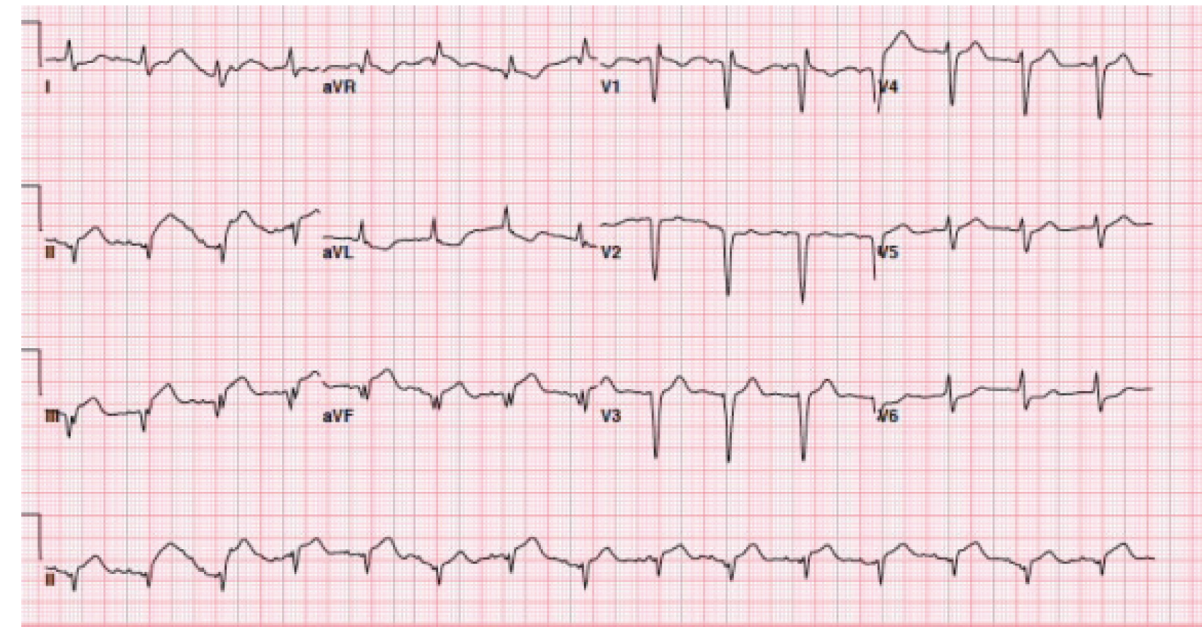

FIgURE 2: ECG after seizure showing acute interval development of ST elevations at leads II, III, and aVF.

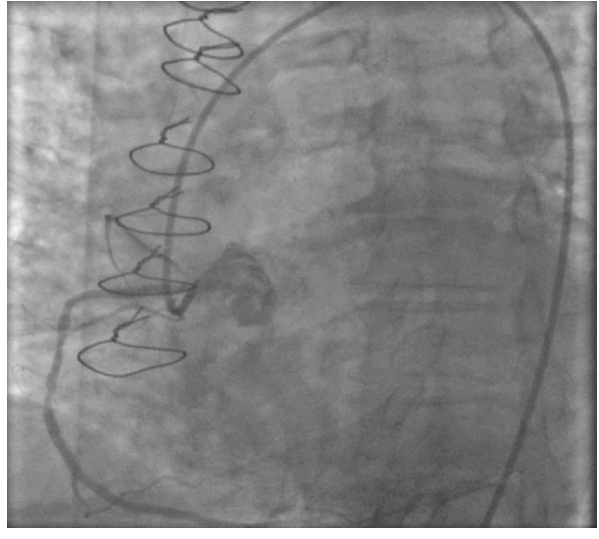

FIGURE 3: Mid-right coronary artery lesion.

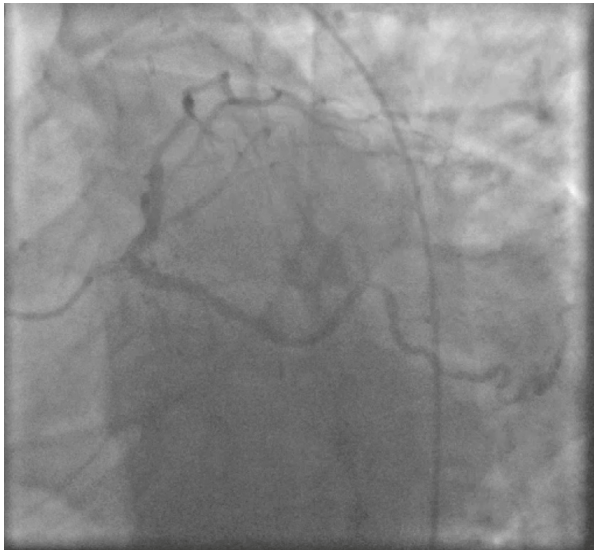

FIGURE 4: Left system angiography. pressure was $187 / 80 \mathrm{mmHg}$. A bedside limited echocardiogram showed EF 50\% with hypokinesis in the basal and mid inferior walls and inferolateral walls. The $2.5 \mathrm{~mm}$ acute ST elevations in inferior leads were persistent for 40 minutes, and later she was transferred to the University Hospitals Cleveland
Medical Center for primary PCI. Her coronary angiography showed a chronically occluded mid-LAD, a mid-LCx with a patent stent, and an $80 \%$ in-stent restenosis in the mid-RCA (RCA) at the site of prior intervention with TIMI flow 3 before intervention (Figures 3 and 4). Optical coherence 


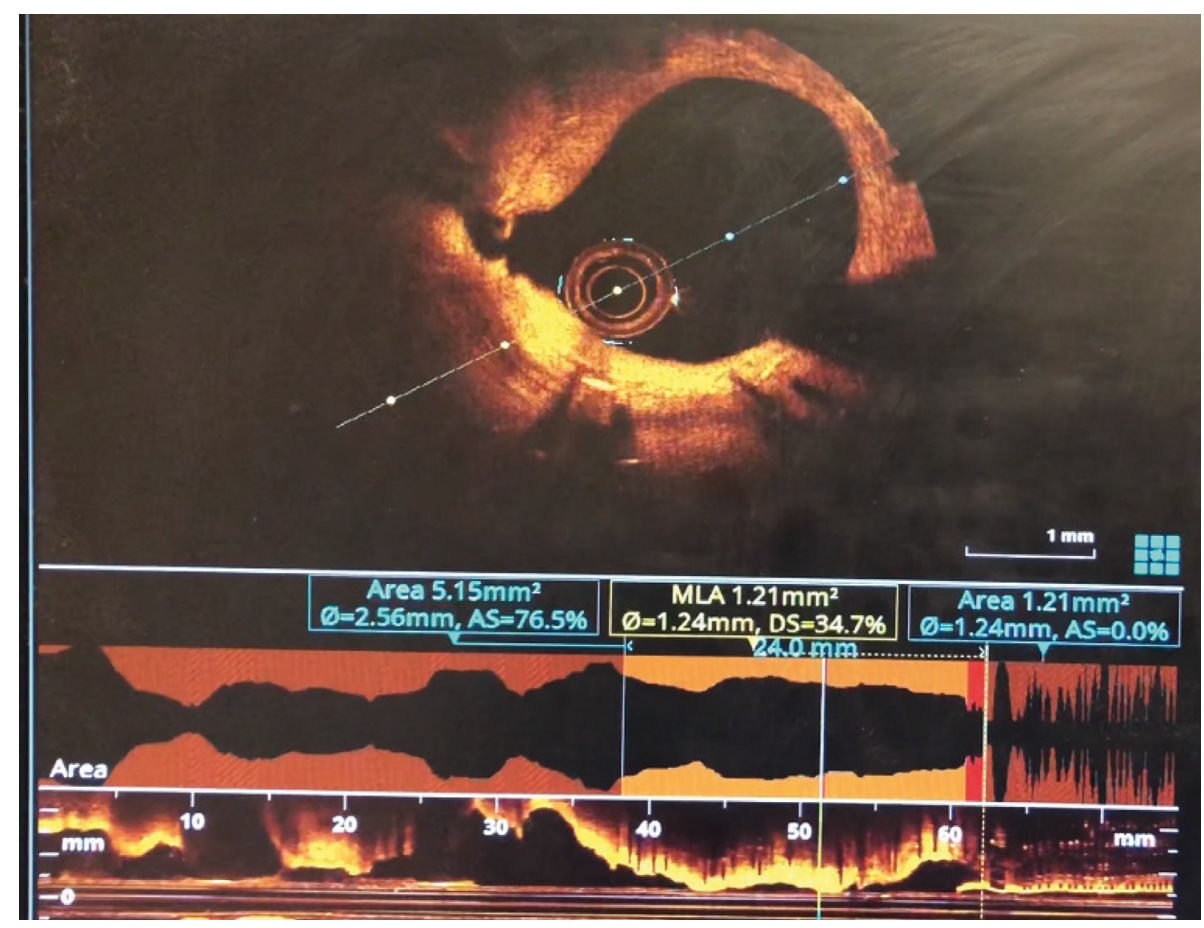

FIgURe 5: Optical coherence tomography.

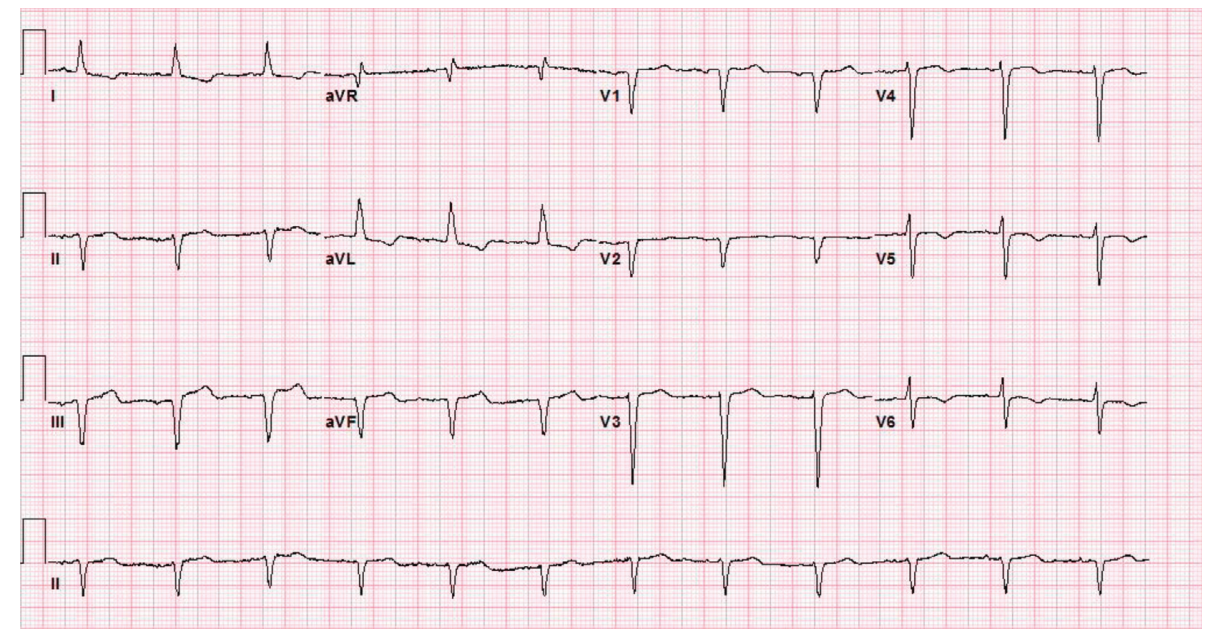

FIgURE 6: ECG after PCI showing marked resolution of ST elevation in leads II, III, and aVF.

tomography (OCT) (Figure 5) revealed malapposition of the prior overlapping stent and no thrombus or plaque rupture. She received a $2.5 \times 38 \mathrm{~mm}$ Xience DES of her mid-RCA stenotic lesion, with good flow after intervention. Postprocedure ECG showed resolution of the ST segment elevation (Figure 6). The next day troponin I was $29.86 \mathrm{ng} / \mathrm{ml}$ and $16.76 \mathrm{ng} / \mathrm{ml}$ twelve hours apart. A complete transthoracic echo on day 3 after STEMI showed left ventricular ejection fraction of $50-55 \%$ and persistent basal and mid inferior wall and inferolateral wall hypokinesis. The patient's mental status remained altered and took two days to return to her baseline after PCI. The neurologist's main impression was posterior reversible encephalopathy syndrome (PRES) secondary to malignant hypertension causing seizures. She was discharged on beta-blockers, dual antiplatelet agents, amlodipine, and statin along with her other medications.

\section{Discussion}

Acute coronary syndrome is typically used to encompass a spectrum ranging from unstable angina, non-ST elevation myocardial infarction, and ST elevation myocardial infarction (STEMI) where the postulated mechanism involves plaque erosion or rupture which this patient did not have $[1,6-8]$. This case shows that acute ST elevation on the ECG can occur without acute plaque erosion or rupture in the setting of seizure. ACh provocation was not performed to test for vasospasm. We postulate seizure-induced 
hypercatecholaminergic-mediated coronary vasospasm at the mid-RCA as the cause of the STEMI. Prior reports of acute ST elevation in noncardiac conditions such as subarachnoid hemorrhage and cocaine abuse postulate acute catecholamine surge causing cardiac myocyte and diffuse coronary vasospasm as the mechanism, especially at the sites of atherosclerosis $[4,9,10]$. The malapposition could have been identified and corrected if OCT had been utilized at the time of her prior intervention. Optical coherence tomography (Figure 5) is useful both to guide intervention and to understand pathophysiological mechanisms in patients with acute ST elevation on the ECG. ECG monitoring is important in patients with acute neurological conditions $[11,12]$.

\section{Conclusions}

Acute seizures can cause acute STEMI in the absence of plaque rupture or erosion. Optical coherence tomography is helpful to discern this type from others like plaque rupture or coronary artery dissection. Acute catecholamine surgemediated vasospasm can be postulated as the cause of STEMI in this patient.

\section{Conflicts of Interest}

The authors declare that there are no conflicts of interests regarding the publication of this paper.

\section{References}

[1] D. M. Kolansky, "Acute coronary syndromes: morbidity, mortality, and pharmacoeconomic burden," American Journal of Managed Care, vol. 15, no. 2, pp. S36-S41, 2009.

[2] E. Arbustini, B. Dal Bello, P. Morbini et al., "Plaque erosion is a major substrate for coronary thrombosis in acute myocardial infarction," Heart, vol. 82, no. 3, pp. 269-272, 1999.

[3] P. Bhat, A. Dretler, M. Gdowski, R. Ramgopal, and E. D. Williams, The Washington Manual of Medical Therapeutics, Washington University, Saint Louis, MO, USA, 2016.

[4] M. A. Samuels, "The brain-heart connection," Circulation, vol. 116, no. 1, pp. 77-84, 2007.

[5] W. B. Bailey and B. R. Chaitman, "Electrocardiographic changes in intracranial hemorrhage mimicking myocardial infarction," New England Journal of Medicine, vol. 349, no. 19, pp. 1874-1875, 2003.

[6] A. Kumar and C. P. Cannon, "Acute coronary syndromes: diagnosis and management," Part I. Mayo Clinic Proceedings, vol. 84, no. 10, pp. 917-938, 2009.

[7] J. Qiao and M. C. Fishbein, "The severity of coronary atherosclerosis at sites of plaque rupture with occlusive thrombosis," Journal of the American College of Cardiology, vol. 17, no. 5, pp. 1138-1142, 1991.

[8] J. Mann and M. J. Davies, "Mechanisms of progression in native coronary artery disease: role of healed plaque disruption," Heart, vol. 82, no. 3, pp. 265-268, 1999.

[9] C. R. Benedict and A. B. Loach, "Sympathetic nervous system activity in patients with subarachnoid hemorrhage," Stroke, vol. 9, no. 3, pp. 237-244, 1978.

[10] E. A. Schenk and A. J. Moss, "Cardiovascular effects of sustained norepinephrine infusions. II. Morphology," Circulation Research, vol. 18, no. 5, pp. 605-615, 1966.
[11] E. A. Osborn and F. A. Jaffer, "Imaging atherosclerosis and risk of plaque rupture," Current Atherosclerosis Reports, vol. 15, no. 10, 2013.

[12] D. Stamper, N. J. Weissman, and M. Brezinski, "Plaque characterization with optical coherence tomography," Journal of the American College of Cardiology, vol. 47, no. 8, pp. C69-C79, 2006. 


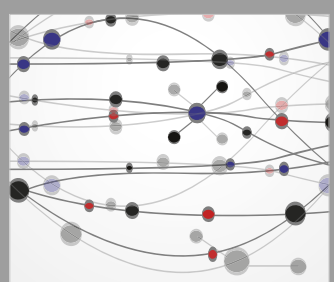

The Scientific World Journal
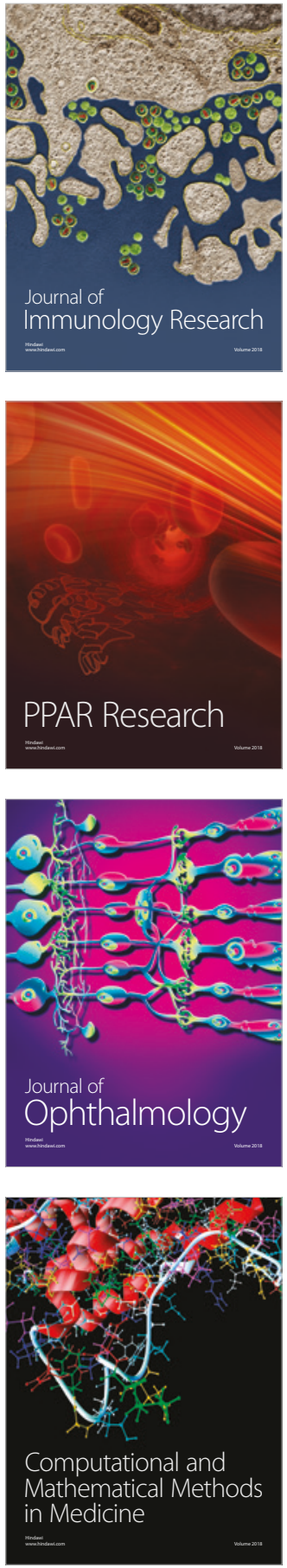

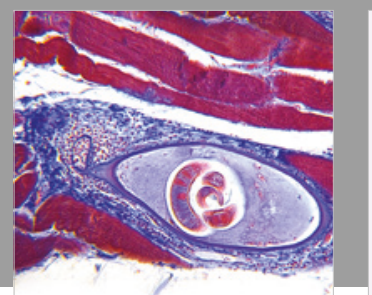

Gastroenterology Research and Practice

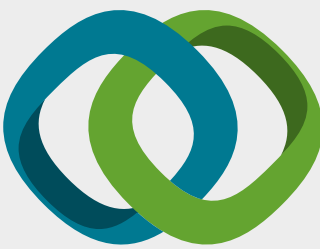

\section{Hindawi}

Submit your manuscripts at

www.hindawi.com
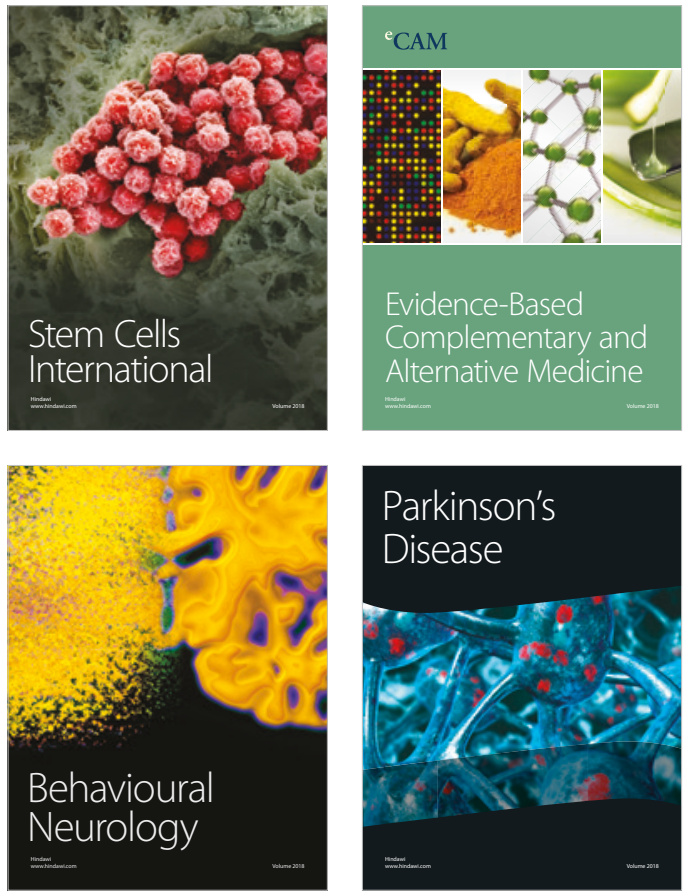

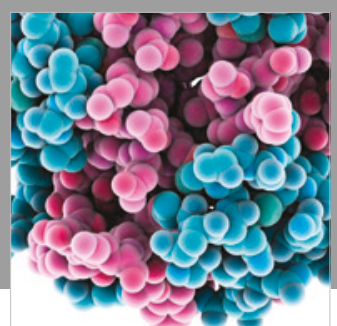

ournal of

Diabetes Research

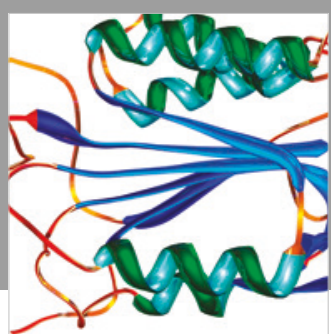

Disease Markers
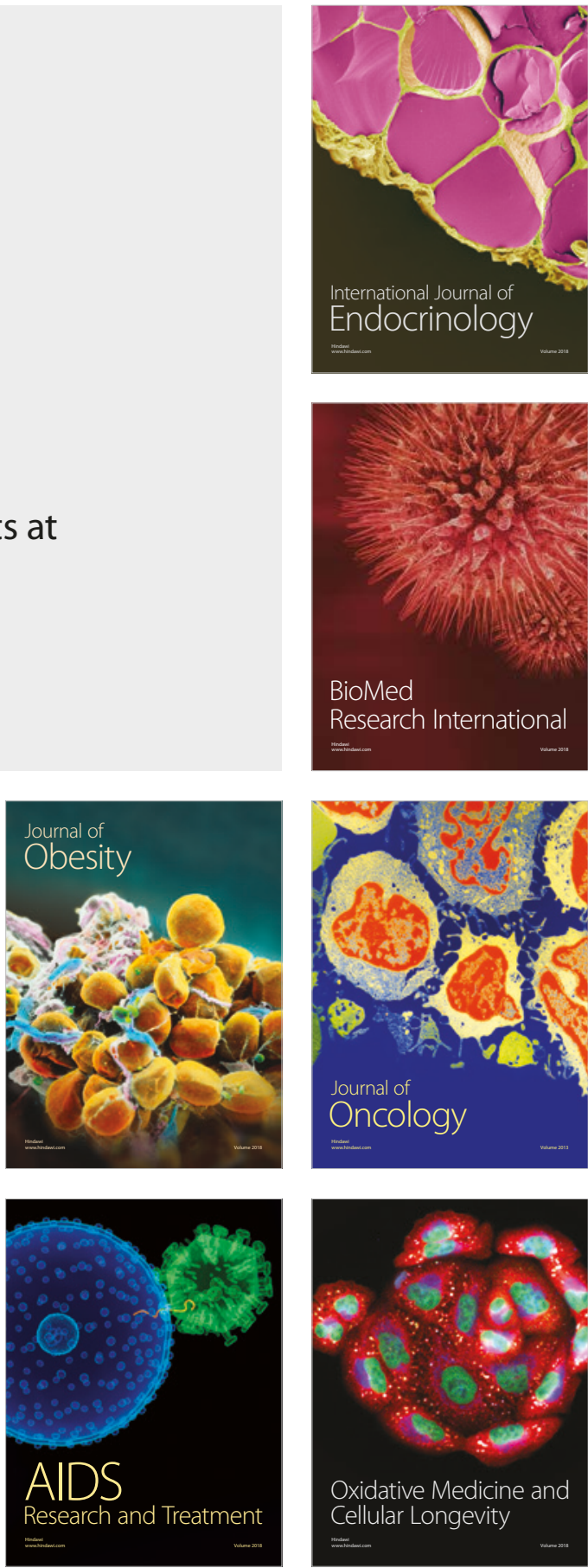